\title{
«Da gäbe es noch etwas zu entdecken»
}

Seit der Gründung im Jahr 2001 war Christoph Rehmann-Sutter Präsident der Nationalen Ethikkommission (NEK) im Bereich der Humanmedizin. Ende März 2009 ist er von seinem Amt zurückgetreten und hat den Ruf an die Universität Lübeck als Professor für Theorie und Ethik der Biowissenschaften angenommen. Ein Kommissionsmitglied bezeichnete ihn als bemerkenswerten Präsidenten, der die Dinge vorantrieb und sensible Themen zu einem Ergebnis führte. Im Gespräch mit der SÄZ gibt er eine Einschätzung seiner Amtszeit, berichtet über Erfolge sowie Schwierigkeiten und gewährt einen Einblick in die Motive seines Wechsels.

Interview:

Annette Eichholtz
Wer auf einen Berufsabschnitt zurückblickt, erinnert sich an Erfreuliches und weniger Erfreuliches. Was fällt Ihnen zu diesen beiden Polen ein?

Das Erfreuliche war ganz bestimmt die kollegiale und freundschaftliche Atmosphäre innerhalb der Kommission, bei allen Differenzen inhaltlicher Art. Die Arbeit war ständig eine Bereicherung. Ich habe mich immer auf die Sitzungen gefreut und ging immer angeregt wieder nach Hause.

Und was hätte besser sein können?

Ich habe es mir einfacher vorgestellt, die Ressourcen zu sichern. Das Budget war von Anfang an zu bescheiden dotiert. Wir mussten z. B. auf die schnelle Übersetzung in alle Landessprachen verzichten, was unschön ist.

Was waren die wichtigsten Erfolge Ihrer Arbeit?

Es ist immer die Frage, was man als Erfolg definiert. Ich sehe zwei Ebenen. Ein Erfolg ist auf den ersten Blick, wenn die Empfehlungen der Kommission umgesetzt werden, und zwar so, wie wir es empfohlen haben. In diesem Sinne gibt es sicher den Erfolg bei der Stammzellenforschung. Wir hatten sicher auch einen nicht zu unterschätzenden Einfluss bei der Präimplantationsdiagnostik (PID), bei der das Parlament in unserem Sinn beschlossen hat, ein Gesetz ausarbeiten zu lassen. Hier waren vorher mehrere Anläufe gescheitert. Oft hatten wir aber nicht direkt messbare Erfolge, sondern indirekte, und das ist die zweite Ebene. Wir konnten eine öffentliche Debatte anregen oder in einem Thema neue Schwerpunkte setzen, wodurch wiederum Politiker, Behörden und Parlament involviert wurden. Da ist die Suizidbeihilfe zu nennen. Hier gab es eine Debatte und über fünf Jahre einen Dialog mit Regierung und Parlament. Und jetzt wird im Justizministerium versucht, Sorgfaltskriterien für Sterbehilfeorganisationen in einen Gesetzesentwurf zu fassen. Aber vorher war eigentlich die Debatte der Erfolg.

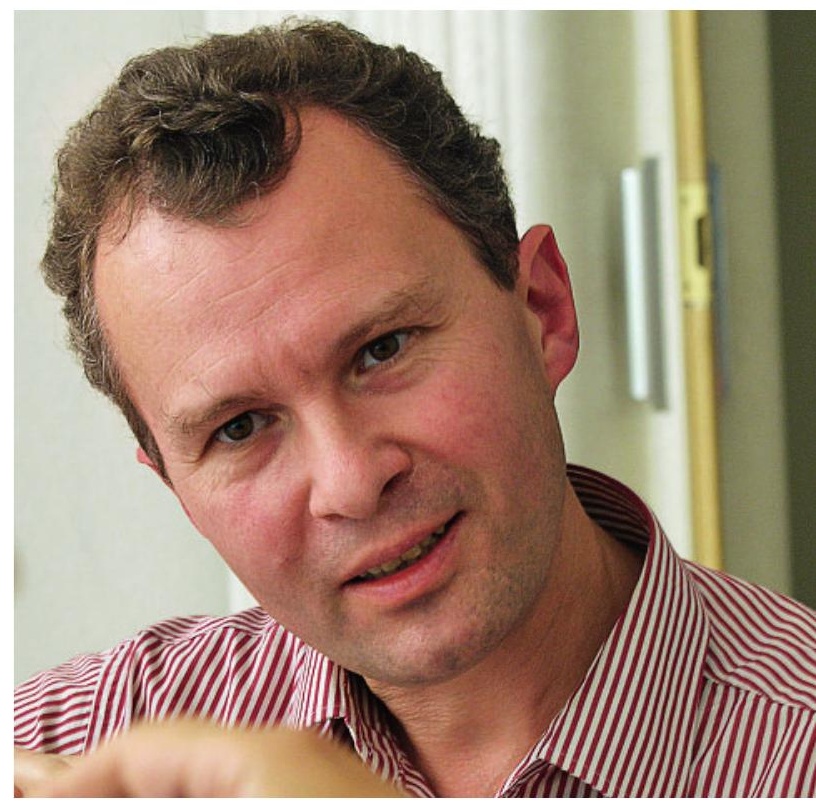

Christoph Rehmann-Sutter erinnert sich gerne an die kollegiale Atmosphäre in der Nationalen Ethikkommission.

Man könnte dennoch den Eindruck haben, dass am Anfang die Empfehlungen fast vollständig in die Gesetzgebung übernommen wurden, bei neueren Themen jedoch nicht mehr. Der Gesetzesentwurf zur PID bleibt deutlich hinter den Vorschlägen der Kommission zurück. Hat diese an Einfluss verloren?

Ich glaube nicht, aber die Themen sind auch unterschiedlich gelagert. Der Erfolg ist wie gesagt nicht immer die Überzeugung der Entscheidungsträger, sondern die Bereicherung der Debatte. Beim PID-Gesetz müssen wir schauen, was letztlich beschlossen wird. Es gibt einen durch Vorsicht gekennzeichneten bundesrätlichen Gesetzesvorschlag, der unserer Meinung nach übervorsichtig und teilweise nicht praktikabel ist, aber jetzt läuft die Debatte. 
Auch bei der Suizidbeihilfe hält die Debatte an. Sie fordern mehr staatliche Kontrolle. Warum, und wie soll das gehen? Die Organisationen bieten eine Technik an, wie man sich schmerzlos und sicher das Leben nehmen kann, und dies mit einer gewissen Professionalisierung. Dadurch treten Hindernisse wie Schuld und Verantwortung, die sich ergeben, wenn man einen Angehörigen um Suizidbeihilfe bittet, weniger auf. Die Hemmschwelle, organisierte Suizidbeihilfe in Anspruch zu nehmen, ist geringer. Die Organisationen berufen sich auf das Prinzip der Autonomie, worunter sie verstehen, den geäusserten Willen anzunehmen. Sie hinterfragen den Willen vielleicht zu wenig. So besteht das Risiko, im Abklärungsprozess zu wenig nach Alternativen zum Tod zu suchen. Respekt für den selbstbestimmten Willen ist wichtig, aber ein Sterbewunsch hat eine Geschichte, und es ist auch die Fürsorge wichtig. Fürsorge kann heissen, sich zum Advokaten des Patienten zu machen und gemeinsam nach Möglichkeiten zu suchen, wie Leben wieder sinnvoll wird. Vielleicht steht die Person unter Druck, und es könnte ihr geholfen werden, indem man etwas an ihrer Situation verändert.

\section{«Oft hatten wir aber nicht direkt messbare Erfolge, sondern indirekte»}

Wie kann das der Staat regeln?

Der Staat kann das im Einzelfall nicht regeln, aber er muss minimale Sorgfaltskriterien bei der Abklärung einfordern können. Dazu braucht es eine Aufsicht über die Organisationen. Momentan gibt es keine solchen Regeln. Es gibt ja auch den sog. Sterbetourismus, und ich finde es schon unter diesem Aspekt gegenüber den Herkunftsländern nicht verantwortbar, keine hinreichenden und transparenten Regeln zu haben.

Suizidbeihilfe ist erlaubt. Entsteht dadurch für Ärzte eine Art moralischer Druck oder Verpflichtung, sie anzubieten? Es muss eine Gewissensentscheidung des einzelnen Arztes, der einzelnen Ärztin bleiben, in einer speziellen, sonst ausweglosen Situation eines Patienten, keine Pflicht. Vielleicht macht er oder sie es für diesen Patienten jetzt nicht, aber für einen anderen schon. Es gibt keinen Anspruch auf ärztliche Suizidbeihilfe.

Herr Couchepin behauptet, die NEK käme immer zu dem Ergebnis, was modern sei, sei auch gut. Was sagen Sie dazu? Da wehre ich mich, denn es stimmt einfach nicht. Unsere Stellungnahmen zeigen deutlich, dass wir nicht einfach die Modernitätsschiene gefahren sind und ethische Bedenken beiseite geräumt haben. Die Bedenken dürfen aber nicht bloss rückwärts gewandt sein. Das konservative Ethikbild, das da mitschwingt, teile ich nicht. Eine medizinische Innovation ist immer zuerst einmal moralisch fragwürdig, im besten Sinn des Wortes frag-würdig. Man muss fragen, ob sie den Menschen gut tut, ob sie zu etwas Gutem für die Gesellschaft führt. Und man muss nach Risiken und Missbrauchsmöglichkeiten fragen. Aber dass wir z. B. die PID deshalb befürwortet hätten, weil sie modern ist, das stimmt überhaupt nicht. Wir haben sie befürwortet, weil sie eine Möglichkeit ist, Leiden zu verhindern von Paaren, die ohne PID einen Schwangerschaftsabbruch erleben, also einen viel schwerwiegenderen Eingriff.

Bundesrat Couchepin hat auch gesagt, die Nationale Ethikkommission sei ein «Problem». Was hat er damit wohl gemeint, und könnte es an dieser kritischen Haltung liegen, dass die Berufung Ihres Nachfolgers so lange gedauert hat? Dass es länger ging, kann auch andere Gründe haben. Aber ich habe den Sinn seines Vorwurfes zu verstehen versucht und habe auch mit Herrn Couchepin darüber gesprochen. Ein Stück weit verstehe ich das Bedenken. Es besteht darin, dass allein das Vorhandensein einer Ethikkommission den Politikern die Aufgabe nehmen könnte, sich selber diese Fragen zu stellen. Sie lesen vielleicht nur noch das «executive summary» des Kommissionsberichts und denken, sie müssten jetzt nicht mehr selber über Ethik nachdenken. Das soll so nicht sein. Aber es geht doch darum, die Ethikkommission richtig zu benützen. Wir sind keine Verantwortungsdelegation, Verantwortung kann man nicht delegieren. Aber wir können Politikern eine Vorarbeit liefern, damit ihre eigenen Entscheidungsprozesse auf einer anderen Ebene beginnen und differenzierter sind.

Haben Sie sich manchmal als ethisches Feigenblatt gefühlt, während Politiker dann doch machen, was aus anderen Gründen opportun ist?

Eigentlich nicht. Aber es passiert natürlich, dass Politiker sagen «die Ethikkommission hat das auch gesagt», um ihr eigenes Anliegen zu stützen. Manchmal werden auch nur Bruchstücke zitiert, aber das ist ja nicht Missbrauch. Es ist eine gewisse Instrumentalisierung, ja, aber das gehört zum Spiel.

Haben Sie eine Idealvorstellung von der Funktion einer Ethikkommission?

Oh ja, meine Idealvorstellung ist eine wache Kommission, eingebettet in einen gesellschaftlichen Entscheidungsprozess, natürlich ausdifferenziert in Regierung, Parlament, Institutionen, medizinische Akademien, Spitäler, in die ganze Hierarchie von Entscheidungsebenen. Und die NEK ist ein Raum der engagierten, interdisziplinär-philosophischen Reflexion darüber, worum es bei der Entwicklung der Medizin letztlich gehen müsste, konkret und mit Einbezug der praktischen Erfahrungen aus den verschiedenen relevanten Bereichen. Sie hilft, einen gemeinsam getragenen Entscheid in ethisch-moralisch heiklen Fragen zu ermöglichen, in einer offenen Weise. Das ist mein Idealbild. 


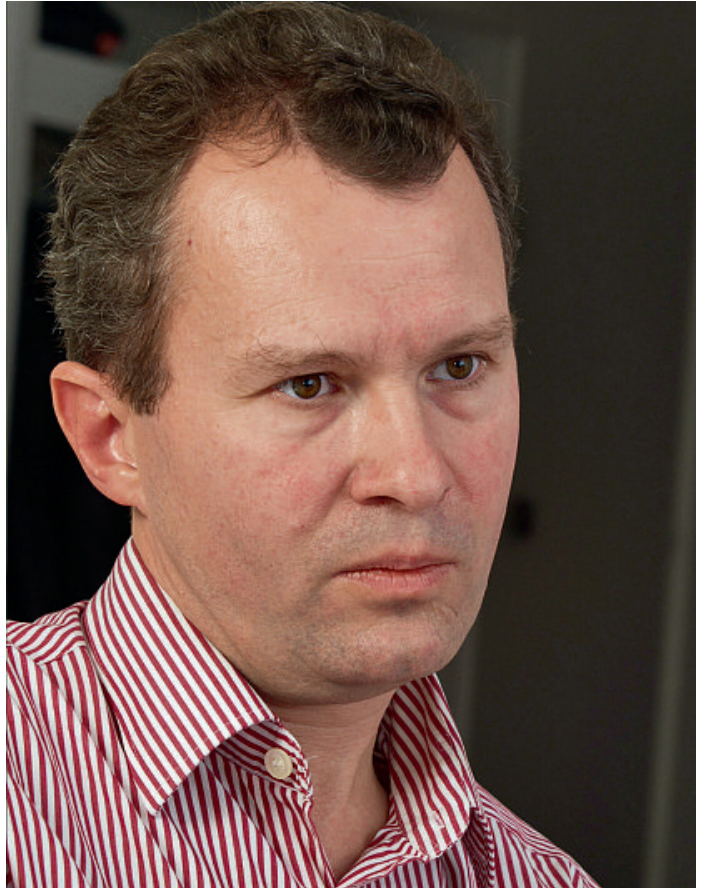

«Dass wir z. B. die PID deshalb befürwortet haben, weil sie modern ist, das stimmt überhaupt nicht»

Wie unterscheidet sich die Realität davon?

Die Kommission muss sich auf relativ wenige Themen beschränken, das heisst, sie kann nicht anders, als selektiv zu sein. Die Fallpauschalen sind z. B. erst jetzt ein Thema. Es war aber ein wichtiger politischer Entscheid schon früher gefallen, sogar eine ziemlich radikale Wende im Gesundheitswesen, aber nur einzelne Spezialisten beschäftigten sich damit. Die NEK hat sich vorgewagt, um die nötige Öffentlichkeit für diese Thematik zu erzeugen.

Sie befürchten Nachteile für die Patientenversorgung durch die DRGs. Kann man Nachteile vermeiden?

Einige Fachleute sagen, sie lassen sich vielleicht vermeiden. Unser Vorschlag ist, dass man von Anfang an mit der nötigen Skepsis und Genauigkeit schaut, was die Einführung der DRGs tatsächlich bewirkt und dies aus Sicht der Patienten und der verschiedenen Berufsgruppen im Spital, z. B. des Caring Teams. Wie gut können sich Ärzte mit Patienten verständigen, sind Gespräche möglich, gibt es genügend Zeit? Eine Begleitforschung muss diese qualitativen Aspekte miterfassen. Und damit soll schon vor Einführung der DRGs begonnen werden, damit der Einführungseffekt beobachtet werden kann. Wo Probleme auftreten, müssen Wege zum «Reparieren» des Systems gesucht werden. Die DRG-Entwickler sagen, das System solle selbstlernend sein. Nimmt man diesen Ansatz ernst genug, dann könnte es vielleicht gut gehen. Das DRGPrinzip, nicht einfach jeden Aufwand zu entschädigen, sondern als Anreiz eine gewisse Pauschale zu setzen, ist ökonomisch gut gedacht, kehrt aber die Interessenlage in den Spitälern um.

\section{Findet die Begleitforschung denn statt?}

Ich habe erfahren, dass es Projektanträge gibt. Dass sie auch finanziert werden, hoffe ich. Ich möchte anregen, Gelder, die in die Umstellung des Systems hineingepumpt werden und aus dem System heraus entstehen, auch für die Begleitforschung zu verwenden. Man sollte nicht die sonst knappen Forschungsmittel belasten.

Wo sollte man denn beim Sparen im Gesundheitssystem ansetzen?

Ein Ansatz sind die Preise, ein anderer ist, die umfassenden Bedürfnisse der Patienten ins Zentrum zu stellen und anzuerkennen, dass sie nicht immer Maximalbehandlung möchten. Sie können aber schwer nein sagen, wenn der Arzt vorschlägt, man könne dies und das noch versuchen. Oft sind grosse Belastungen damit verbunden, iatrogenes Leiden. Wenn man die Entscheidungsfindung mehr darauf abstellt, was dem Patienten wirklich gut tut, nicht nur auf Lebensverlängerung, dann würde es wohl weniger Behandlungen geben, vor allem am Lebensende.

Das wird ja gerade diskutiert. Braucht es dafür nicht Richtlinien? Wann bietet man eine teure Chemotherapie noch an, wann nicht? Wer entscheidet das?

Da müssten schon die Patienten das letzte Wort haben. Und es kommt sehr darauf an, wie man eine Behandlung empfiehlt. Wenn der Arzt sagt: «Wir können das schon noch machen, das schaffen Sie», dann kommt halt eine Zustimmung heraus.

Sie sehen das in der Verantwortung von Arzt und Patient, es wäre kein Thema für die Ethikkommission?

Nein, da geht es um den Einzelfall. Ich hätte Bedenken, wenn man sie vom Patienten und von der Ärztin wegnimmt. Eine Ausnahme sind Konfliktfälle und Unklarheiten. Aber da kann nicht eine nationale Kommission einspringen, dafür braucht es am Ort klinisch-ethische Konsilien.

Gespart wurde auch bei der NEK. Wird die Budgetkürzung deren Arbeit sehr erschweren?

Es waren von Anfang an zu wenig Mittel, und es hat sehr weh getan, dass es noch weniger wurden. Ich denke, die Kommission ist schon funktionsfähig, aber man könnte mehr aus dieser Institution machen. In Deutschland etwa gehört der Ethikrat zum Stolz des Landes und in Frankreich kommt manchmal sogar der Staatspräsident. Einen solchen Enthusiasmus für eine NEK habe ich in der Schweiz noch nicht gespürt. Da gäbe es noch etwas zu entdecken. 
War die Budgetkürzung mit ein Grund für Ihren Rücktritt? Oder wollen Sie die Gründe nicht verraten?

Doch, es gab ja einen ganz praktischen Grund. Die Kürzung hat mich persönlich betroffen. Meine 40 Stellenprozente und damit ein Teil meines Lebensunterhalts wurden weggenommen und in eine drei Viertel kleinere Entschädigung umgewandelt. Als ich dann nach Lübeck berufen wurde, war mir klar, dass ich von dort aus die Schweizer Ethikkommission nicht würde leiten können. Ausserdem fand ich auch, dass frischer Wind gut tut und der Zeitpunkt für einen Wechsel jetzt, nach acht Jahren, günstig ist. Man soll nicht am Sessel kleben, bis es nicht mehr gut läuft.
Sie haben zuerst Molekularbiologie studiert und hätten sicher eine gute Stelle gefunden. Warum haben Sie dann noch Philosophie und Soziologie, eher brotlose Künste, studiert?

Weil es mich einfach unerhört interessiert hat, die philosophischen Fragen an der Molekularbiologie haben mich nicht mehr losgelassen. Es ist ein kulturell und philosophisch so herausfordernder Prozess, der sich heute in der modernen Biologie abspielt. Auch die Interaktion mit ökologischen Problemen hat mich sehr beschäftigt. Beides sind Menschheitsfragen. Dazu wollte ich einen Beitrag leisten, so gut ich kann. Philosophie und Soziologie brauchte ich als Handwerkszeug,

\section{«Unser Vorschlag ist, dass man von Anfang an mit der nötigen Skepsis und Genauigkeit schaut, was die Einführung der DRGs tatsächlich bewirkt»}

Möchten Sie Ihrem Nachfolger Otfried Höffe einen Rat geben?

Er ist ein hochkarätiger Autor und Gelehrter. Die NEK hat durch ihn viel gewonnen, und ich gratuliere ihm zur Wahl. In meiner Zeit habe ich gelernt, dass die Öffentlichkeitsdimension für die Arbeit der NEK ganz besonders anspruchsvoll ist. Sie darf nicht nur darauf schauen, brillant geschriebene Papers zu haben, die sie dann unter die Leute bringt, sondern muss die Menschen ausserhalb der Kommission in ihre Prozesse involvieren. Dazu ist es wichtig, dass der Präsident öffentlich präsent und ansprechbar ist. Es kommt darauf an, wie er als Figur im gesundheitspolitischen Raum sichtbar wird und dass er auch kritisierbar ist. Die Kommission muss nicht lehren, sondern zuerst selbst lernen. Als lernende Kommission muss sie vorangehen. Das verlangt, dass sie wahrnimmt, zuhört. Sie soll auch eine zuhörende Kommission sein. Dann hat sie Glaubwürdigkeit in der Öffentlichkeit, und diese Glaubwürdigkeit ist das sine qua non, um Politikberatung zu betreiben. um auch Forschung betreiben zu können. Ich habe gehofft, dass ich irgendwie damit werde leben können, weil das Thema Bioethik einfach gesellschaftlich wichtig ist.

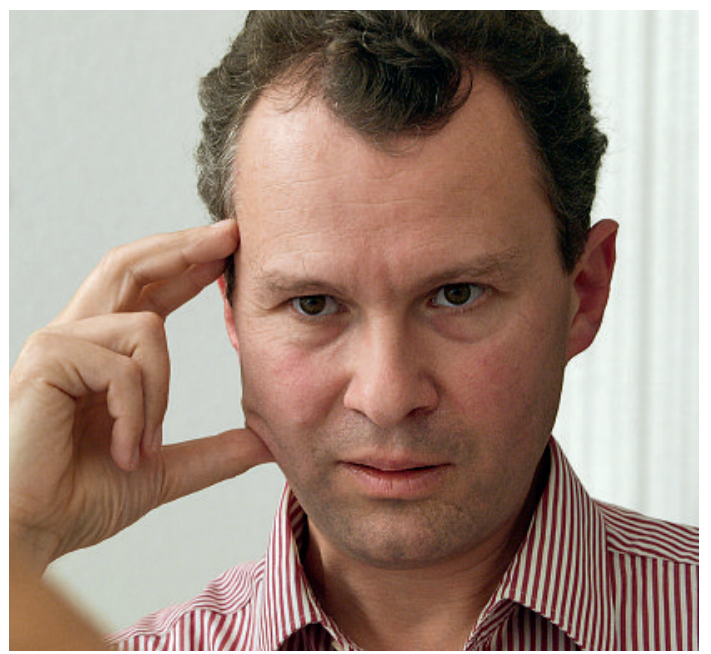

